\title{
Lack of M30 expression correlates with factors reflecting tumor progression in rectal cancer with preoperative chemoradiotherapy
}

\author{
SUSUMU SAIGUSA, YASUHIRO INOUE, KOJI TANAKA, YOSHINAGA OKUGAWA, YUJI TOIYAMA, \\ KEIICHI UCHIDA, YASUHIKO MOHRI and MASATO KUSUNOKI
}

Department of Gastrointestinal and Pediatric Surgery, Mie University Graduate School of Medicine, Tsu, Mie 514-8507, Japan

Received July 2, 2013; Accepted September 12, 2013

DOI: $10.3892 / \operatorname{mco} .2013 .189$

\begin{abstract}
Preoperative chemoradiotherapy (CRT) is an effective tool for local control that functions by inducing cancer cell apoptosis and inhibiting cell growth. The aim of this study was to evaluate the expression of caspase-cleaved keratin 18 cytoskeletal protein, M30, which is known as an apoptotic marker in residual rectal cancer following preoperative CRT. A total of 72 patients with rectal cancer who had undergone preoperative CRT were enrolled in this study. Immunostaining with M30 cytodeath antibody was performed and the correlation between M30 staining and clinicopathological variables was analyzed. Furthermore, we examined the correlation of M30 staining with the expression of Bax, Bcl-2, Ki67 and PCNA using transcriptional and immunohistochemical analyses. The results showed that 34 (47\%) patients were positive for M30 staining. Lack of M30 expression was significantly correlated with advanced $\mathrm{T}$ stage, postoperative stage and tumor recurrence $(\mathrm{P}<0.05)$. Patients with M30 staining had better recurrence-free survival (RFS) than those without it $(\mathrm{P}=0.0301)$. In the immunohistochemical analysis, residual cancer cells with M30 staining lacked Ki67 expression. No significant correlation was observed between M30 positivity and the gene expression of apoptotic and proliferative markers. In conclusion, findings of the present study suggested that the evaluation of M30 expression may be useful in the prediction of tumor recurrence in rectal cancer patients who have been treated with preoperative CRT.
\end{abstract}

\section{Introduction}

Rectal cancer is one of the most common types of cancer in Japan and other developed nations. The introduction of preoperative chemoradiotherapy (CRT) and total mesorectal

Correspondence to: Dr Susumu Saigusa, Department of Gastrointestinal and Pediatric Surgery, Mie University Graduate School of Medicine, 2-174 Edobashi, Tsu, Mie 514-8507, Japan E-mail: saigusa@clin.medic.mie-u.ac.jp

Key words: apoptosis, M30, preoperative chemoradiotherapy, rectal cancer, tumor recurrence excision (TME) for the management of locally advanced rectal cancer has decreased local recurrence rates and increased patient survival (1-3). However, disease recurrence remains the major cause of mortality in rectal cancer patients. Identification of predictors for disease recurrence and poor prognosis would aid in the successful treatment of these patients. Predictive pathological and molecular biomarkers for revelaing the prognosis for patients with rectal cancer following preoperative CRT have been reported $(4,5)$. Several studies have shown that the tumor regression grade (TRG) or a pathological response following preoperative CRT are predictors of clinical outcomes (6-9). Moreover, the apoptotic and proliferative status has been reported to be associated with tumor recurrence and poor prognosis $(10,11)$. Radiotherapy is an effective tool for local control that functions by inducing cancer cell apoptosis and death and inhibiting cell growth in various malignancies.

The anti-M30 monoclonal antibody reacts with a caspase-cleaved product of keratin 18, a type I intermediate filament protein and major component of single-layer and glandular epithelial cells, which only detects apoptotic events in tumor or normal epithelial cells (12). Although terminal deoxyribonucleotidyl transferase dUTP nick end-labeling (TUNEL) is a common method used for the detection of DNA fragmentation resulting from the apoptotic signaling cascade it has also been used to evaluate apoptotic cell death, the anti-M30 antibody is considered to be a promising alternative to the TUNEL assay for the detection of apoptotic cells (13). Previous studies have shown that the apoptotic status of cancer cells following CRT using M30 immunohistochemistry was not associated with local recurrence and prognosis, although the level of apoptosis in primary tumors without pretreatment predicted a high risk of local recurrence and a poor prognosis for rectal cancer patients $(10,14-16)$. The studies mentioned previously have suggested that the predisposition to intrinsic apoptosis prior to CRT was more important than therapy-induced apoptosis for local recurrence and prognosis. However, we hypothesized that therapy-induced apoptosis is not present at the time of evaluation due to the interval between CRT and surgery, and that the residual cancer cells possess intrinsic or spontaneous apoptotic potential.

The aim of this retrospective study was to evaluate residual rectal cancer cells following preoperative CRT by $\mathrm{M} 30$ immunostaining, in addition to investigating the 
correlation of M30 expression with clinical outcomes and the expression of apoptotic and proliferative markers.

\section{Materials and methods}

Patients and specimens. From 2001 to 2011, 77 patients with rectal cancer received preoperative CRT followed by surgery at Mie University Hospital (Mie, Japan). The criteria used for the induction of preoperative CRT were that patients were required to i) be $\leq 80$ years of age; ii) be diagnosed as clinical stage II/III based on the International Union Against Cancer TNM classification, with no evidence of distant metastases; iii) exhibit no invasion of the external sphincter muscle or elevator muscle of the anus; and iv) show no evidence of deep venous thrombosis. In total five patients exhibited a complete pathological response and were excluded from participation in this study. The study design was approved by Mie University Hospital's Ethics review board. All patients signed informed consent forms to allow for the use of their tissues in this study.

5-Fluorouracil (5-FU)-based CRT regimen. Patients with rectal cancer were treated with short-course (a dose of 20 Gy in 4 fractions) or long-course (a dose of $45 \mathrm{~Gy}$ in 25 fractions) of radiotherapy using the 4-field box technique with concurrent chemotherapy to take advantage of 5-FU radiosensitization. Patients underwent concurrent pharmacokinetic modulation chemotherapy [5-FU administered by intravenous infusion: $600 \mathrm{mg} / \mathrm{m}^{2}$ for $24 \mathrm{~h}$, and tegafur-uracil (UFT) administered orally: $400 \mathrm{mg} / \mathrm{m}^{2}$ for 5 days] (17). Short-course radiotherapy at the Mie University Hospital is different from standard short-course radiotherapy, which consists of a dose of $25 \mathrm{~Gy}$ in 5 fractions. The present regimen was designed as described previously for several reasons: a biologically equivalent dose (BED) of $20 \mathrm{~Gy}$ in 4 fractions was calculated using a linear quadratic model, and its BED was $30 \mathrm{~Gy}(\alpha / \beta$ ratio, $10 \mathrm{~Gy})$, which had sufficient efficacy in reducing the local failure of radiotherapy (18). Furthermore, the reduction of postoperative complications, such as ileitis induced by preoperative radiotherapy, were considered. In total 50 patients received short-course radiotherapy with chemotherapy over 1 week. The remaining 22 patients received long-course radiotherapy with chemotherapy for 4 weeks. The time interval between preoperative CRT and surgery was 2-3 weeks for short-course and 4-6 weeks for long-course irradiation patients. The patients underwent standard surgery, including TME, and received 5-FU-based adjuvant chemotherapy following surgery for between 6 months to 1 year.

Clinical and pathological response to CRT. The clinical response following preoperative CRT was evaluated by a barium enema, endoscopy, computed tomography and magnetic resonance imaging. The clinical response was subsequently graded as a complete response, a partial response, no change or progressive disease. The TRG was evaluated using the 3-point Ryan TRG system (19). The 3-point Ryan system combines TRG1 (no viable cancer cells) and TRG2 (single cells or small groups of cancer cells) to form one category: 3-point TRG1. TRG3 (residual cancer outgrown by fibrosis) forms the 3-point TRG2, and TRG4 (significant fibrosis outgrown by cancer) and TRG5 (no fibrosis with extensive residual cancer) are combined in order to form the 3-point TRG3.

Immunohistochemistry for M30, Bax, Bcl-2, Ki67 and PCNA. Immunohistochemistry was performed as described previously (20). Anti-M30 CytoDeath (mouse monoclonal antibody, clone M30; dilution 1:100; Peviva AB, Bromma, Sweden), polyclonal rabbit anti-human Bax (code A3533; dilution 1:2000; DakoCytomation, Carpinteria, CA, USA), monoclonal mouse anti-human Bcl-2 oncoprotein (clone 124; dilution 1:100; DakoCytomation), monoclonal mouse anti-human Ki-67 antigen (clone MIB-1; used as supplied; DakoCytomation), monoclonal mouse anti-proliferating cell nuclear antigen (PCNA; clone PC10; dilution 1:200; DakoCytomation) antibodies were used as primary antibodies. Negative controls were run simultaneously. M30 positivity was identified as brown cytoplasmic staining.

Immunohistochemical evaluation of M30. The number of cytoplasmic M30 positive cells in residual cancer cells per five fields at a magnification of X100 was counted under a light microscope (BX50, Olympus, Tokyo, Japan). Each sample was evaluated in a blinded manner by two investigators who had no clinical or pathological information regarding the origin of the samples.

Quantitative polymerase chain reaction ( $q P C R$ ). Microdissection of FFPE, cDNA synthesis, and qPCR were performed as described previously (20). Primers for $B A X$, $B C L 2, M K I 67, P C N A, G A P D H$ and ACTB ( $\beta$-actin) were designed with Primer3 software (Biology Workbench Version 3.2; San Diego Super Computer Center, University of California, San Diego, CA, USA). The sequences used were as follows: $B A X$-specific primers, sense: CTTTGCCAGCAAAC TGGTG, antisense: CAGCCCATGATGGTTCTGA; $B C L 2$-specific primers, sense: TCGCCCTGTGGATGACTGA, antisense: CAGAGACAGCCAGGAGAAATCAA; MKI67-specific primers, sense: TGAGCCTGTACGGCTAAA ACA, antisense: TTGACTTCCTTCCATTCTGAAG; PCNA-specific primers, sense: GAAGCACCAAACCAGGA GAA, antisense: TATCGGCATATACGTGCAAA; GAPDH, sense: GGAAGGTGAAGGTCGGAGTC, antisense: AATGAAGGGGTCATTCATGG; and ACTB, sense: ACAGAGCCTCGCCTTTGC, antisense: GCGGCGATAT CATCATCC. Relative mRNA levels were determined by the standard curve method. Standard curves and line equations were generated using 5 -fold serially diluted solutions of cDNA from the colon cancer cell line, Lovo or Human Reference Total RNA (Clontech Laboratories, Inc., Mountain View, CA, USA). Target gene expression was calculated using the standard curve and quantitative normalization of cDNA in each sample was performed using the expression of the ACTB gene as an internal control.

Statistical analyses. Statistical analyses were performed using Stat View 5.0 for Windows (SAS Institute Inc., Cary, NC, USA). The values of each target gene are expressed as the median values in tables. Significant differences were analyzed using the Chi-square test. The associations between continuous and categorical variables were evaluated using the Mann-Whitney 
Table I. Patient characteristics and the correlation of M30 staining with clinicopathological variables.

\begin{tabular}{|c|c|c|c|c|}
\hline Variables & No. $(\%)$ & M30-negative $(n=38)$ & M30-positive $(n=34)$ & P-value \\
\hline \multicolumn{5}{|l|}{ Gender } \\
\hline Male & $54(75)$ & 29 & 25 & \multirow[t]{2}{*}{0.785} \\
\hline Female & $18(25)$ & 9 & 9 & \\
\hline \multicolumn{5}{|l|}{ Age (median; 64) } \\
\hline$<64$ & $36(50)$ & 21 & 15 & \multirow[t]{2}{*}{0.345} \\
\hline$\geq 64$ & $36(50)$ & 17 & 19 & \\
\hline \multicolumn{5}{|l|}{ ypT classification } \\
\hline $1 / 2$ & $27(38)$ & 10 & 17 & \multirow[t]{2}{*}{0.038} \\
\hline $3 / 4$ & $45(62)$ & 28 & 17 & \\
\hline \multicolumn{5}{|l|}{ ypN classification } \\
\hline Absent & $47(65)$ & 21 & 26 & \multirow[t]{2}{*}{0.059} \\
\hline Present & $25(35)$ & 17 & 8 & \\
\hline \multicolumn{5}{|l|}{ Postoperative stage } \\
\hline $\mathrm{I} / \mathrm{II}$ & $44(61)$ & 18 & 26 & \multirow[t]{2}{*}{0.011} \\
\hline III/IV & $28(39)$ & 20 & 8 & \\
\hline \multicolumn{5}{|l|}{ Lymphatic invasion } \\
\hline Absent & $18(25)$ & 9 & 9 & \multirow[t]{2}{*}{0.785} \\
\hline Present & $54(75)$ & 29 & 25 & \\
\hline \multicolumn{5}{|l|}{ Vascular invasion } \\
\hline Absent & $36(50)$ & 15 & 21 & \multirow[t]{2}{*}{0.059} \\
\hline Present & $36(50)$ & 23 & 13 & \\
\hline \multicolumn{5}{|l|}{ Histology } \\
\hline Well/moderate & $63(88)$ & 32 & 31 & \multirow[t]{2}{*}{0.372} \\
\hline Poor/signet/mucinous & $9(12)$ & 6 & 3 & \\
\hline \multicolumn{5}{|l|}{ TRG } \\
\hline Grade 1 & $16(23)$ & 7 & 9 & \multirow[t]{3}{*}{0.684} \\
\hline Grade 2 & $37(51)$ & 21 & 16 & \\
\hline Grade 3 & $19(26)$ & 10 & 9 & \\
\hline \multicolumn{5}{|l|}{ Radiotherapy } \\
\hline Short (20 Gy/4 fractions) & $50(69)$ & 25 & 25 & \multirow[t]{2}{*}{0.477} \\
\hline Long (45 Gy/25 fractions) & $22(31)$ & 13 & 9 & \\
\hline \multicolumn{5}{|l|}{ Recurrence } \\
\hline Absent & $53(74)$ & 24 & 29 & \multirow[t]{2}{*}{0.030} \\
\hline Present & $19(26)$ & 14 & 5 & \\
\hline
\end{tabular}

TRG, tumor regression grade. Chi-square test.

$\mathrm{U}$ test for the two groups. Recurrence-free survival (RFS) and overall survival (OS) times were calculated from the date of surgery to the date of disease recurrence or patient death, respectively. RFS and OS probabilities were calculated using the Kaplan-Meier product limit method, and intergroup differences were determined using a log-rank test. $\mathrm{P}<0.05$ was considered to indicate a statistically significant difference.

\section{Results}

Patient and tumor characteristics. The median age of the study subjects was 64 years (range, 35-77 years) and the male:female ratio was 5:2 (Table I). Post-CRT pathological T stages were
ypT0 $(n=1)$, pT1 $(n=7)$, ypT2 $(n=19)$, ypT3 $(n=43)$ and ypT4 $(n=2)$ (Table I). In total, 25 patients $(35 \%)$ presented with lymph node metastases. Sixty-three tumors ( $88 \%$ ) had well- or moderately differentiated adenocarcinoma histology. Local recurrence alone was exhibited in 4 patients $(5.5 \%)$, while 15 patients $(20.8 \%)$ had distant recurrence. Patterns of distant recurrence were observed as both liver and lung metastases in 2 patients, lung metastasis alone in 9 patients, liver metastasis alone in 1 patient and peritoneal metastasis in 2 patients. The median follow-up period was 62 months (range, 9-147 months).

Correlation between M30 staining and clinicopathological variables. There were 34 patients (47\%) with M30 staining 
Table II. Expression of M30 and proliferative and apoptotic markers.

\begin{tabular}{lccr}
\hline Gene & M30-positive $(\mathrm{n}=21)$ & M30-negative $(\mathrm{n}=29)$ & P-value \\
\hline BAX & 0.807 & 0.743 & 0.7161 \\
BCL2 & $1.13 \times 10^{-4}$ & 0.000 & 0.3247 \\
PCNA & 0.018 & 0.022 & 0.7854 \\
MKI67 & $14.4 \times 10^{-6}$ & $3.8 \times 10^{-6}$ & 0.8483 \\
\hline
\end{tabular}

$\mathbf{A}$

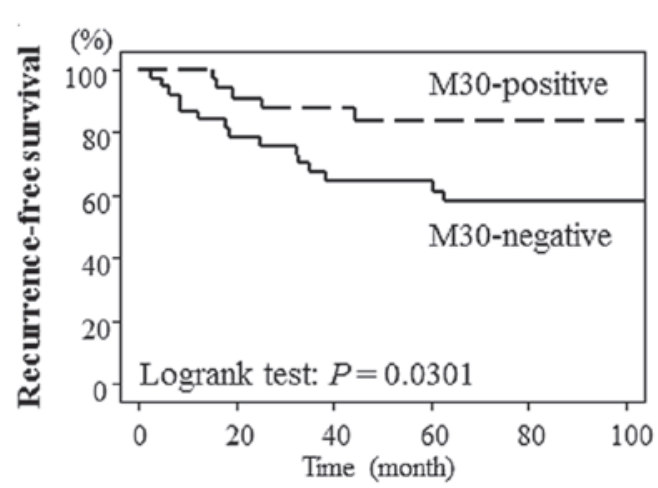

B

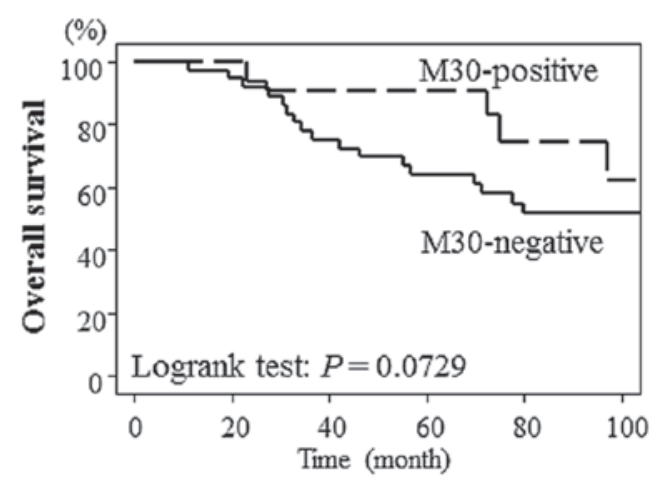

Figure 1. Kaplan-Meier curves showing the correlation between M30 positivity and survival. (A) Recurrence-free survival. (B) Overall survival.

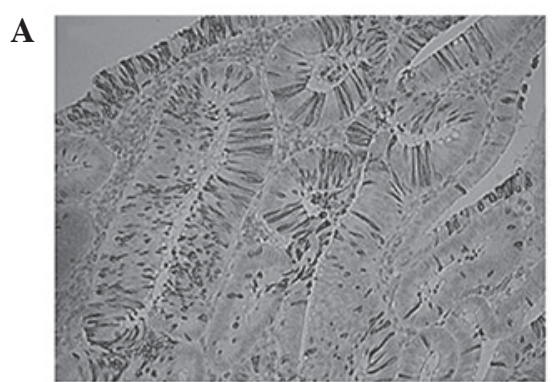

B
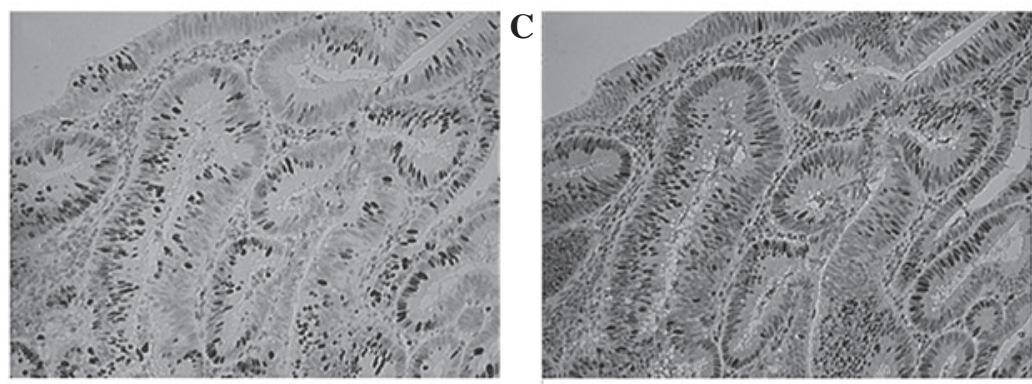

D
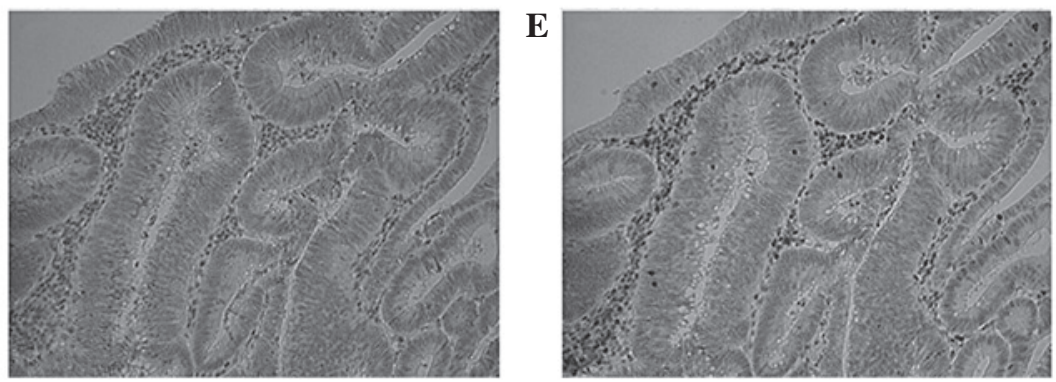

Figure 2. Immunohistochemical staining of (A) M30, (B) Ki67, (C) PCNA, (D) Bax and (E) Bcl-2. Original magnification, x200.

(range of percentage of M30-positive cancer cells, 0-32\%). The mean percentage of cancer cells with M30 staining was particularly low, $2.93 \%$. Therefore, patients were divided into two groups according to M30 positivity. Table I shows the correlation of M30 staining with clinicopathological variables. Patients without M30 staining of residual rectal cancer following CRT more frequently had advanced ypT classification, advanced postoperative stage, and tumor recurrence $(\mathrm{P}=0.038,0.011$ and 0.030 , respectively). M30 positivity was correlated with lymph node metastasis and vascular invasion, despite the lack of significant differences $(\mathrm{P}=0.059$ and
0.059, respectively). We observed no correlation between M30 staining and other clinicopathological findings, including TRG.

Lack of M30 staining was associated with poor RFS. Fig. 1 shows the survival curve for RFS and OS according to M30 positivity using the Kaplan-Meier method. Lack of M30 staining was significantly associated with poorer RFS $(\mathrm{P}=0.0301)$. Furthermore, there was a tendency to have an improved prognosis in patients with positive positive M30 staining compared with patients lacking M30 staining $(\mathrm{P}=0.0729)$. 
Immunohistochemical findings for M30, Bax, Bcl-2, Ki67 and PCNA. The expression of apoptotic markers, Bax and Bcl-2, and proliferative markers, Ki67 and PCNA were examined, in cases with high M30 expression. Residual cancer cells with M30 staining lacked Ki67 and PCNA expression. However, there was no correlation between M30 staining and apoptotic markers (Fig. 2).

Correlation of M30 staining with BAX, BCL2, MKI67 and $P C N A$ expression. In the transcriptional analysis, there was no correlation between M30 staining and the expression of $B A X$, BCL2, MKI67 or PCNA (Table II).

\section{Discussion}

In this study, the immunohistochemical evaluation of M30 for apoptotic status correlated with low $\mathrm{T}$ stage and tumor recurrence in patients with locally advanced rectal cancer subsequent to preoperative CRT. Furthermore, we observed that patients with M30 staining demonstrated improved RFS. The results of this study are different to those of other studies in that the apoptotic status following preoperative CRT was not associated with tumor recurrence or a poor prognosis (10,14-16). One reason for this discrepancy is that the analyses performed in the present study utilized M30 positivity compared with previous studies which divided groups by the mean of median values of M30-positive cells. Adell et al (21) demonstrated that the mean percentage of apoptotic cells was $1.1 \%(0-14.5 \%)$ in rectal cancer following preoperative radiotherapy using the TUNEL method. Koornstra et al (22) reported that the mean percentage of cancer cells with M30 staining was $2 \%$ in primary colorectal cancer. The results of those studies suggested that there were a small number of apoptotic cancer cells in primary and pretreated rectal cancer. In this study, the mean percentage of cancer cells with M30 staining was $2.93 \%$. Therefore, we divided the patients into two groups according to M30 positivity. Results obtained from the pathological response demonstrated no significant correlation between M30 staining and TRG. This may have been due to the fact that TRG is determined by quantifying the proportion of residual cancer cells relative to the stroma with fibrosis and vasculopathy of the entire tumor bed, and does not reflect therapy-induced apoptosis of residual cancer cells. Furthermore, M30 is expressed during early apoptosis except for caspase-independent apoptosis and mitotic catastrophe (12). In this study, M30 expression likely represents spontaneous apoptosis rather than therapy-induced apoptosis due to the time intervals between preoperative CRT and surgery, and cancer cells induced by apoptosis are replaced by fibrosis. However, de Bruin et al (14) reported that M30-positive cells were increased by radiotherapy, suggesting that radiotherapy induced apoptosis.

We also investigated whether there was any correlation between the apoptotic status evaluated by M30 and the expression of apoptotic and proliferative markers. In the transcriptional analysis, we observed no significant correlation between M30 staining and the gene expression of BAX, BCL2 MKI67 and $P C N A$. However, the gene expression of $M K I 67, P C N A$ and $B C L 2$ correlated with the pathological response following CRT (data not shown). This suggests that TRG reflects the proliferative activity of residual cancer cells following CRT. Results of the immunohistochemical evaluation of M30, Bax, Bcl-2, Ki67 and PCNA revealed that M30-positive cells lacked Ki67 expression. The Ki67 protein is present during all active phases of the cell cycle, with the exception of the G0 phase. This finding confirmed that M30 staining detected cell death. By contrast, the expression of Bax, Bcl-2, and PCNA did not correlate with M30 staining. Bcl-2 and Bax regulate caspase activation. Bcl-2 is an important inhibitor of apoptosis, while the overexpression of Bax induces apoptosis (23). The M30 cytodeath antibody recognizes a neo-epitope formed following the caspase cleavage of keratin 18 and detects apoptosis of an epithelial origin. Therefore, the difference in apoptotic status determined between M30 and these apoptotic markers may result in no significant correlation between M30 and the apoptotic markers.

In conclusion, the evaluation of M30 expression is useful in the prediction of tumor recurrence in rectal cancer patients treated with preoperative CRT, although it is unknown as to whether the apoptosis detected by M30 staining was intrinsic or induced by therapy. However, the data in this study should be interpreted with caution. A significant limitation of this study was the small number of patients studied $(n=72)$, particularly those with recurrence $(n=19)$, and the retrospective nature of the study. This study also included two neoadjuvant radiation regimens with different time intervals between pretreatment and surgery. Furthermore, our short-course regimen deviated from the standard method. Therefore, a larger study population, long-term follow-up and unification of pretreatments are required in order to validate the results.

\section{Acknowledgements}

The authors would like to thank Motoko Ueeda, Yuka Kato and Chihiro Hibi for providing excellent technical assistance.

\section{References}

1. Sauer R, Becker H, Hohenberger W, et al: Preoperative versus postoperative chemoradiotherapy for rectal cancer. N Engl J Med 351: 1731-1740, 2004.

2. van den Brink M, Stiggelbout AM, van den Hout WB, et al: Clinical nature and prognosis of locally recurrent rectal cancer after total mesorectal excision with or without preoperative radiotherapy. J Clin Oncol 22: 3958-3964, 2004.

3. Guillem JG, Chessin DB, Cohen AM, et al: Long-term oncologic outcome following preoperative combined modality therapy and total mesorectal excision of locally advanced rectal cancer. Ann Surg 241: 829-838, 2005.

4. Smith FM, Reynolds JV, Miller N, et al: Pathological and molecular predictors of the response of rectal cancer to neoadjuvant radiochemotherapy. Eur J Surg Oncol 32: 55-64, 2006.

5. Bertolini F, Bengala C, Losi L, et al: Prognostic and predictive value of baseline and posttreatment molecular marker expression in locally advanced rectal cancer treated with neoadjuvant chemoradiotherapy. Int J Radiat Oncol Biol Phys 68: 1455-1461, 2007.

6. Rodel C, Martus P, Papadoupolos T, et al: Prognostic significance of tumor regression after preoperative chemoradiotherapy for rectal cancer. J Clin Oncol 23: 8688-8696, 2005.

7. Bujko K, Michalski W, Kepka L, et al: Association between pathologic response in metastatic lymph nodes after preoperative chemoradiotherapy and risk of distant metastases in rectal cancer: an analysis of outcomes in a randomized trial. Int $\mathbf{J}$ Radiat Oncol Biol Phys 67: 369-377, 2007.

8. Dhadda AS, Dickinson P, Zaitoun AM, et al: Prognostic importance of Mandard tumour regression grade following pre-operative chemo/radiotherapy for locally advanced rectal cancer. Eur J Cancer 47: 1138-1145, 2011.

9. Topova L, Hellmich G, Puffer E, et al: Prognostic value of tumor response to neoadjuvant therapy in rectal carcinoma. Dis Colon Rectum 54: 401-411, 2011. 
10. Tannapfel A, Nusslein S, Fietkau R, et al: Apoptosis, proliferation, bax, bcl-2 and p53 status prior to and after preoperative radiochemotherapy for locally advanced rectal cancer. Int $\mathrm{J}$ Radiat Oncol Biol Phys 41: 585-591, 1998.

11. Rupa JD, de Bruine AP, Gerbers AJ, et al: Simultaneous detection of apoptosis and proliferation in colorectal carcinoma by multiparameter flow cytometry allows separation of high and low-turnover tumors with distinct clinical outcome. Cancer 97: 2404-2411, 2003.

12. Leers MP, Kolgen W, Bjorklund V, et al: Immunocytochemical detection and mapping of a cytokeratin 18 neo-epitope exposed during early apoptosis. J Pathol 187: 567-572, 1999.

13. Caulin C, Salvesen GS and Oshima RG: Caspase cleavage of keratin 18 and reorganization of intermediate filaments during epithelial cell apoptosis. J Cell Biol 138: 1379-1394, 1997.

14. de Bruin EC, van de Velde CJ, van de Pas S, et al: Prognostic value of apoptosis in rectal cancer patients of the Dutch total mesorectal excision trial: radiotherapy is redundant in intrinsically high-apoptotic tumors. Clin Cancer Res 12: 6432-6436, 2006.

15. de Heer P, Gosens MJ, de Bruin EC, et al: Cyclooxygenase 2 expression in rectal cancer is of prognostic significance in patients receiving preoperative radiotherapy. Clin Cancer Res 13: 2955-2960, 2007.

16. Gosens MJ, Dresen RC, Rutten HJ, et al: Preoperative radiochemotherapy is successful also in patients with locally advanced rectal cancer who have intrinsically high apoptotic tumours. Ann Oncol 19: 2026-2032, 2008.
17. Yoshikawa R, Kusunoki M, Yanagi H, et al: Dual antitumor effects of 5-fluorouracil on the cell cycle in colorectal carcinoma cells: a novel target mechanism concept for pharmacokinetic modulating chemotherapy. Cancer Res 61: 1029-1037, 2001.

18. Colorectal Cancer Collaborative Group: Adjuvant radiotherapy for rectal cancer: a systematic overview of 8,507 patients from 22 randomised trials. Lancet 358: 1291-1304, 2001.

19. Ryan R, Gibbons D, Hyland JM, et al: Pathological response following long-course neoadjuvant chemoradiotherapy for locally advanced rectal cancer. Histopathology 47: 141-146, 2005.

20. Saigusa S, Tanaka K, Toiyama Y, et al: Correlation of CD133, OCT4, and SOX2 in rectal cancer and their association with distant recurrence after chemoradiotherapy. Ann Surg Oncol 16: 3488-3498, 2009.

21. Adell GC, Zhang H, Evertsson S, et al: Apoptosis in rectal carcinoma: prognosis and recurrence after preoperative radiotherapy. Cancer 91: 1870-1875, 2001.

22. Koornstra JJ, Rijcken FE, De Jong S, et al: Assessment of apoptosis by M30 immunoreactivity and the correlation with morphological criteria in normal colorectal mucosa, adenomas and carcinomas. Histopathology 44: 9-17, 2004.

23. Reed JC: Double identity for proteins of the Bcl-2 family. Nature 387: 773-776, 1997. 